\title{
Factors that are Effective in Surgery Preferences of Patients Diagnosed with Breast Cancer who are Admitted to Radiation Oncology Clinics
}

\author{
(D) Sema YILMAZ RAKICI
}

Department of Radiation Oncology, Recep Tayyip Erdoğan University, Faculty of Medicine, Rize-Turkey

\begin{abstract}
OBJECTIVE
In this study, the factors affecting the choice of treatment were investigated in patients diagnosed with breast cancer who were treated with breast-conserving surgery or mastectomy.

\section{METHODS}

Factors that may be effective in surgical choice were aimed to be determined by retrospectively examining patient files regarding age, pathological type, diameter and lateralization of the tumor, number of lymph nodes in the axilla, estrogen and progesterone receptor (ER and PR), c-erbB-2 status, the place of residence, center of operation and distribution of patients according to years. The relationships among the data in this study were examined using statistical methods.
\end{abstract}

\section{RESULTS}

The mean age of the patients was $52.06 \pm 11.91$ (age range: $28-86$ ). Tumor lateralization was the right side in $44.4 \%$ of the patients and the left side in $55.6 \%$ of the patients. There were significantly more modified radical mastectomy (MRM) surgeries in the cases with left-sided lateralization and more breastconserving surgeries $(B C S)$ in the patients with right-sided lateralization ( $\mathrm{p}=0.001)$. Significantly more BCSs were found to be performed when the tumor diameter was less than $2.5 \mathrm{~cm}$, and more MRMs were performed as the nodal stage increased $(\mathrm{p}=0.000, \mathrm{p}<0.001)$. The patients with positive $P R$ receptors were treated with BCS significantly more frequently $(\mathrm{p}=0.005)$. The presence of radiotherapy facilities increased the frequency of BCSs, and the MRM rates were higher in the patients living in rural areas.

\section{CONCLUSION}

We found that the patients with good prognostic characteristics known for breast cancer were more frequently treated with BCSs, whereas the patients with poor prognostic characteristics were more frequently treated with MRMs. We also found that being younger, living in a city, having right-sided lateralization and radiotherapy facilities increased the choice of BCS.

Keywords: Breast-conserving surgery; mastectomy; right/left breast cancer; radiotherapy facilities. Copyright $\odot$ 2019, Turkish Society for Radiation Oncology

\section{Introduction}

There have been some changes in the treatment of breast cancer since the past. Some tumors are considered to have local spread in their behavior, and some other tumors are considered to be systemic from the onset. Therefore, it is emphasized that tumors should be treated with a multidisciplinary approach.[1-4] Today, international consensus guidelines have been developed for the management of breast cancer in patients. [5] The
Received: June 20, 2019

Accepted: August 06, 2019

Online: October 28, 2019

Accessible online at:

www.onkder.org

OPEN ACCESS This work is licensed under a Creative Commons

Attribution-NonCommercial 4.0 International License.
Dr. Sema YILMAZ RAKICI,

Recep Tayyip Erdoğan Üniversitesi,

Tıp Fakültesi,

Radyasyon Onkolojisi,

Rize-Turkey

E-mail: sema.rakici@erdogan.edu.tr 
surgical method to ensure local control has been gaining popularity in the direction from a modified radical mastectomy (MRM) to breast-conserving surgery (BCS).[6] In patients undergoing BCS, body integrity is maintained, and the quality of life is enhanced by ensuring the conservation of the organ. However, various factors, such as patient and tumor characteristics, may be effective in the selection of surgical treatment, and even devices and equipment possessed by the hospital. In this study, various factors that could be effective in treatment selection were investigated in patients who had undergone surgery due to breast cancer, including the opening of the Radiation Oncology Clinic and the city in which the patients lived.

\section{Materials and Methods}

This study included 133 female patients who were admitted to the Radiation Oncology Clinic of our hospital between September 2013 and September 2018 and did not have any distant metastasis, had undergone surgery due to diagnosis of breast cancer and were receiving adjuvant radiotherapy. The patients' age, pathological type, diameter and lateralization of the tumor, number of lymph nodes in the axilla, estrogen and progesterone receptor (ER and PR), c-erbB-2 status, place of residence, the center of operation and distribution were examined according to years. An attempt was made to identify the factors that could be effective in the type of surgery performed on breast cancer patients.

Ethical committee approval and informed consent were not obtained due to the retrospective design of this study. This study was conducted in accordance with the ethical standards of the responsible committee on human experimentation and the Declaration of Helsinki.

\section{Statistical Analyses}

Statistical analysis of the data was carried out using the SPSS Version 17 software. Frequencies, percentages, means and standard deviations and minimum and maximum values were used as descriptive statistics. Ttests and Chi-squared analyses were used as analytical statistics. In the results, $\mathrm{p}<0.05$ was considered to be significant.

\section{Results}

This study included a total of 133 female patients who had undergone MRM or BCS due to breast cancer, all of whom were receiving adjuvant RT. The mean age of the patients was $52.06 \pm 11.91$ (age range: $28-86$ ). Among the patients, 51.9\% (69 patients) had undergone BCS, and $48.1 \%$ (64 patients) had undergone MRM. The mean age at the time of diagnosis was $50.49 \pm 10.77$ (age range: $28-75)$ in the patients undergoing BCS, while it was $53.75 \pm 12.91$ (age range: $28-86$ ) in the patients undergoing MRM. Although the patients undergoing BCS were relatively younger, the age difference was not statistically significant $(\mathrm{p}=0.810)$. Additionally, the median age was found to be 49 in the patients undergoing BCS and 54 in the patients undergoing MRM. Considering the age distribution, the patients undergoing BCS peaked in the range of 40-45, while the patients undergoing MRM peaked in the range of 55-60 years (Fig. 1). The peak age range of the patients undergoing BCS was found to be 15 years earlier.

Tumor lateralization was the right side in $44.4 \%$ of the patients (59 patients) and the left side in 55.6\% (74 patients). More cases with left-sided lateralization (60.8\%) underwent MRMs, while more of the cases with right-sided lateralization (67.8\%) underwent BCSs. The data for tumor lateralization are shown in Table 1. The difference between the types of lateralization (right and left) was statistically significant $(\mathrm{p}=0.001)$.

The mean $\pm S D$ values of the tumor diameters were found to be $2.1580 \pm 1.156(\min -\max =.5 \mathrm{~cm}-7 \mathrm{~cm})$ in the patients undergoing BCS and $3.63 \pm 1.97$ (min$\max =1.1 \mathrm{~cm}-9.4 \mathrm{~cm}$ ) in those undergoing MRM. The tumor diameter was found to be smaller in the patients undergoing BCS. More BCSs (68.9\%) were performed on the patients with a tumor diameter of $<2.5 \mathrm{~cm}$, while more MRMs (62.5\%) were performed on the patients with a tumor diameter of $\geq 2.5 \mathrm{~cm}$ (Table 2 ). The difference in the BCS operations based on tumor diameters of $<2.5 \mathrm{~cm}$ was statistically significant $(\mathrm{p}<0.001)$.

While $80.9 \%$ of the patients who underwent BCS were N0, 19.1\% of the patients who underwent MRM consisted of N0 patients. A total of $16 \mathrm{~N} 3$ patients all underwent MRM. Among the N2 patients, $84.2 \%$ underwent MRM, and $15.8 \%$ underwent BCS. MRMs were preferred for the $\mathrm{N} 2$ and $\mathrm{N} 3$ patients, as well as the N1 patients, whereas BCSs were preferred in the

\begin{tabular}{lccccc} 
Table 1 & \multicolumn{3}{c}{ Tumor lateralization } & & \\
\hline \multirow{2}{*}{ Lateralization } & Right & Count & 40 & 19 & 59 \\
& & $\%$ & $67.8 \%$ & $32.2 \%$ & $100.0 \%$ \\
& Left & Count & 29 & 45 & 74 \\
& & $\%$ & $39.2 \%$ & $60.8 \%$ & $100.0 \%$ \\
& Total & Count & 69 & 64 & 133 \\
& & $\%$ & $51.9 \%$ & $48.1 \%$ & $100.0 \%$ \\
\hline
\end{tabular}




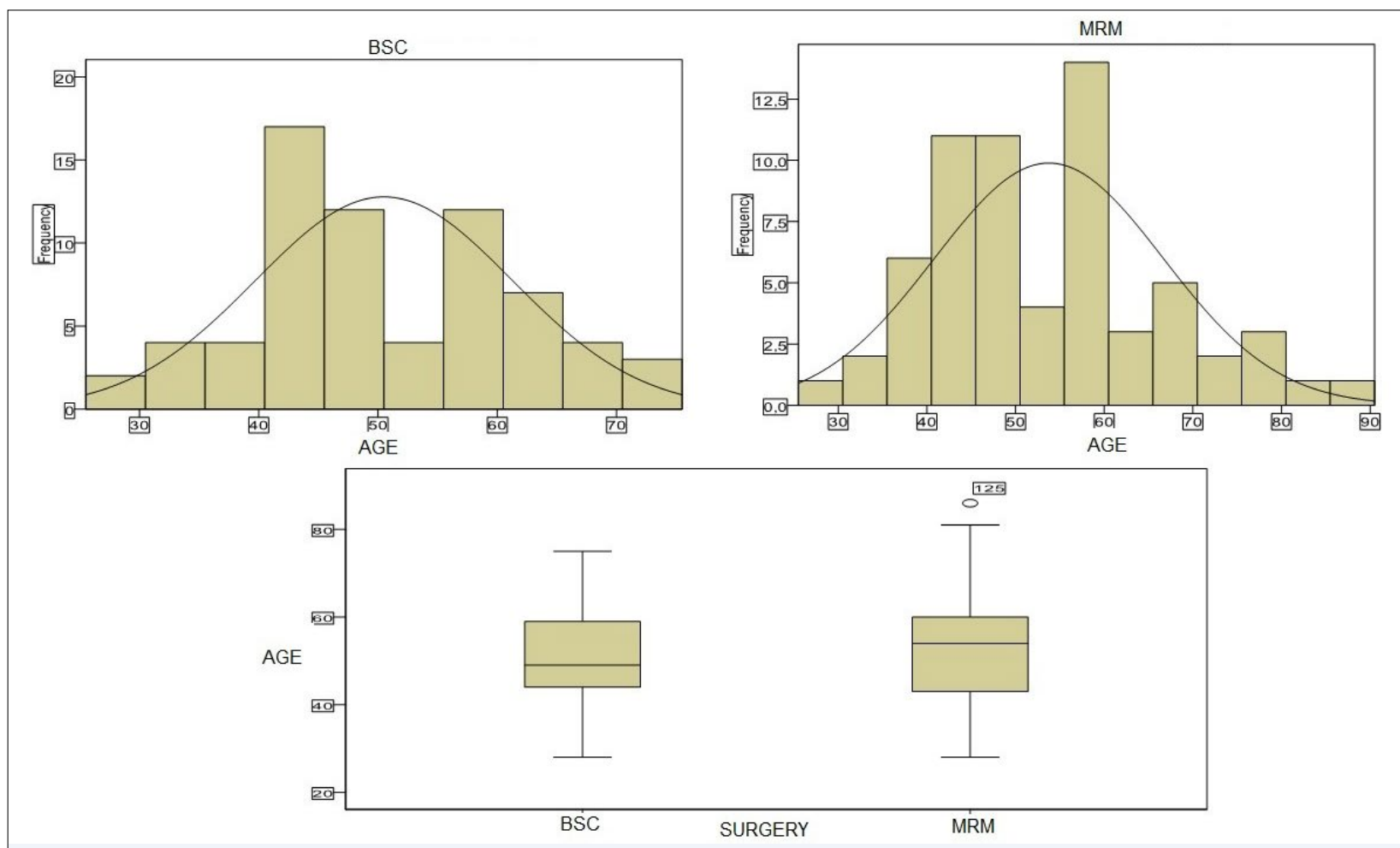

Fig. 1. Age range of the cases undergoing BCS and MRM.

Table 2 The relationship between tumor diameter and surgery

\begin{tabular}{lccccc} 
& & & BSC & MRM & Total \\
\hline Tumor & $<2.5 \mathrm{~cm}$ & Count & 42 & 19 & 61 \\
diameters & & $\%$ & $68.9 \%$ & $31.1 \%$ & $100.0 \%$ \\
& $>=2.5 \mathrm{~cm}$ & Count & 27 & 45 & 72 \\
& & $\%$ & $37.5 \%$ & $62.5 \%$ & $100.0 \%$
\end{tabular}

N0 patients. As the nodal stage increased, the rate of MRMs increased significantly $(\mathrm{p}=0.000, \mathrm{p}<0.001)$.

Among the patients, 75\% (100 patients) were ERpositive, while $25 \%$ (33 patients) were ER-negative. ER positivity was $55.0 \%$ in the patients undergoing BCS, while it was $42.4 \%$ in the patients undergoing MRM. No significant correlation was found between ER positivity and surgery type $(\mathrm{p}=0.292)$. Among the patients, $60 \%$ (80 patients) were PR-positive, while $40 \%$ (53 patients) were PR-negative. PR positivity was found to be $62.5 \%$ in the patients undergoing BCS and $37.5 \%$ in the patients undergoing MRM. $64.2 \%$ of the patients undergoing MRM were composed of PR-negative patients. There was a significant correlation between PR positivity and undergoing BCS. The patients with positive PR receptors were treated with BCS significantly more frequently ( $p=0.005)$. The rate of BCSs in the patients who were c-erbB-2-negative was found to be $54.5 \%$, while the same rate for MRMs was $45.5 \%$. The difference was statistically insignificant ( $\mathrm{p}=0.541)$. The rate of MRMs in patients with c-erbB- $2 \geq 3$ was $65.5 \%$, while the rate of BCSs was $34.5 \%$. A correlation that was nearly significant was found between performing MRMs and having c-erbB- $2 \geq 3(\mathrm{p}=0.056)$. The ER, PR and c-erbB-2 statuses of the patients are shown in Table 3.

\begin{tabular}{|c|c|c|c|c|c|}
\hline & & & BSC & MRM & Total \\
\hline \multirow[t]{4}{*}{ ER } & Positive & Count & 55 & 45 & 100 \\
\hline & & $\%$ & $55.0 \%$ & $45.0 \%$ & $100.0 \%$ \\
\hline & Negative & Count & 14 & 19 & 33 \\
\hline & & $\%$ & $42.4 \%$ & $57.6 \%$ & $100.0 \%$ \\
\hline \multirow[t]{4}{*}{ PR } & Positive & Count & 50 & 30 & 80 \\
\hline & & $\%$ & $62.5 \%$ & $37.5 \%$ & $100.0 \%$ \\
\hline & Negative & Count & 19 & 34 & 53 \\
\hline & & $\%$ & $35.8 \%$ & $64.2 \%$ & $100.0 \%$ \\
\hline \multirow[t]{4}{*}{ c-erbB-2 } & Positive & Count & 33 & 34 & 67 \\
\hline & & $\%$ & $49.3 \%$ & $50.7 \%$ & $100.0 \%$ \\
\hline & Negative & Count & 36 & 30 & 66 \\
\hline & & $\%$ & $54.5 \%$ & $45.5 \%$ & $100.0 \%$ \\
\hline \multirow[t]{2}{*}{ Total } & & Count & 69 & 64 & 133 \\
\hline & & $\%$ & $51.9 \%$ & $48.1 \%$ & $100.0 \%$ \\
\hline
\end{tabular}


Table 4 The centers where the patients were operated on

\begin{tabular}{lccccc} 
& & & BCS & MRM & Total \\
\hline $\begin{array}{lcccc}\text { Operated } \\
\text { center }\end{array}$ & $\begin{array}{c}\text { External } \\
\text { center }\end{array}$ & Count & 41 & 27 & 68 \\
& & $\%$ & $60.3 \%$ & $39.7 \%$ & $100.0 \%$ \\
& Our & Count & 28 & 37 & 65 \\
& hospital & $\%$ & $43.1 \%$ & $56.9 \%$ & $100.0 \%$ \\
& Total & $\begin{array}{c}\text { Count } \\
\end{array}$ & 69 & 64 & 133 \\
& & $\%$ & $51.9 \%$ & $48.1 \%$ & $100.0 \%$
\end{tabular}

In this study, $43.1 \%$ of the patients undergoing BCS were operated at our hospital, while $60.3 \%$ of them were operated outside our hospital. Among the patients undergoing MRM, 56.9\% were operated at our hospital, and $39.7 \%$ of them were operated outside our hospital (Table 4). Significantly more MRM operations were found to be performed at our hospital ( $p=0.047)$.

The rates depending on the patients undergoing BCS living in cities, districts and villages were found to be $47.1 \%, 62.5 \%$ and $38.5 \%$, respectively, while the MRM rates were $52.9 \%, 37.5 \%$ and $61.5 \%$, respectively. The rate of MRMs increased as the place where the patients lived changed from cities to rural areas, but the difference was not statistically significant $(p=0.087)$. When we categorized the place where the patients lived as rural areas (villages) or cities (provincial and district centers) in two separate categories, $19.6 \%$ of the patients (26 patients) lived in rural areas, and $80.4 \%$ (107 patients) lived in cities. Among the patients living in rural areas, $61.5 \%$ of them underwent MRM, and $38.5 \%$ of them underwent BCS. Among the patients living in cities, $55.1 \%$ of them underwent BCS, and $44.9 \%$ of them underwent MRM. Although there were more MRMs in the patients living in rural areas and more BCSs in the patients living in cities, the difference between these was not statistically significant $(p=1.91)$.
Given the patients' places of living concerning tumor diameters and nodal stages, their statuses of living in a city, district or village were not found to be significantly correlated with the increase in tumor diameter and nodal stage $(\mathrm{p}=0.288$ and $\mathrm{p}=0.466$, respectively). The information about the patients' places of living concerning tumor diameters and nodal stages is shown in Table 5 .

Considering the rates of BCSs by years, there was increasingly more BCSs after 2013 until 2018 (from 2013 to 2018 , the rates of BCSs were $36.4 \%, 48.7 \%$, $100.0 \%, 50.0 \%, 72.4 \%$ and $40.0 \%$, respectively). The year 2018 did not reflect the actual number of operations since the year was not over yet during the data collection period. The maximum rate of BCSs was $72.4 \%$ (21 patients) in 2017, and the maximum rate of MRMs was 63.6\% (21 patients) in 2013.

When the distribution of the percentages of the patients was examined according to the provinces where they were admitted to hospitals, Rize ranked first concerning the frequency of admissions with a rate of $69.2 \%$ (92 patients). Among the external admission centers, Artvin ranked first concerning the frequency of admissions with 18.8\% (25 patients), Trabzon ranked second with $3.8 \%$ (5 patients), and Istanbul ranked third with $2.3 \%$ (three patients). Other provinces constituted $0.8 \%$ of the admissions (Sakarya, Ordu, Kırklareli, Erzincan, Çorum, Bursa, Ankara and Amasya). Among the patients admitted in Artvin, 60\% of them underwent BCS, and 40\% underwent MRM. Among the patients admitted in Rize, $48.9 \%$ of them underwent BCS, and $51.1 \%$ of them underwent MRM.

When the pathological types were examined, the findings showed that $74.4 \%$ of the cases (99 patients) had invasive ductal carcinoma (IDC), 4.5\% (six patients) had invasive lobular carcinoma (ILC), and 2.3\% (three patients) had ductal carcinoma in situ (DCIS). Other types were found to be tubular carcinoma in

Table 5 The patients' places of living in relation to tumor diameters and nodal stages

\begin{tabular}{|c|c|c|c|c|c|c|c|c|}
\hline & & & \multicolumn{2}{|c|}{ Tumor diameters } & \multicolumn{4}{|c|}{ Nodal stages } \\
\hline & & & $<2.5 \mathrm{~cm}$ & $>=2.5 \mathrm{~cm}$ & No & N1 & N2 & N3 \\
\hline \multirow[t]{8}{*}{ Living places } & City & Count & 19 & 32 & 21 & 13 & 8 & 9 \\
\hline & & $\%$ & $37.3 \%$ & $62.7 \%$ & $41.2 \%$ & $25.5 \%$ & $15.7 \%$ & $17.6 \%$ \\
\hline & District & Count & 29 & 27 & 34 & 10 & 8 & 4 \\
\hline & & $\%$ & $51.8 \%$ & $48.2 \%$ & $60.7 \%$ & $17.9 \%$ & $14.3 \%$ & $7.1 \%$ \\
\hline & Village & Count & 13 & 13 & 13 & 7 & 3 & 3 \\
\hline & & $\%$ & $50.0 \%$ & $50.0 \%$ & $50.0 \%$ & $26.9 \%$ & $11.5 \%$ & $11.5 \%$ \\
\hline & Total & Count & 61 & 72 & 68 & 30 & 19 & 16 \\
\hline & & $\%$ & $45.9 \%$ & $54.1 \%$ & $51.1 \%$ & $22.6 \%$ & $14.3 \%$ & $12.0 \%$ \\
\hline
\end{tabular}


$4.5 \%$ (six patients), mucinous carcinoma in 3.8\% (five patients), adenoid cystic carcinoma in $0.8 \%$ (one patient), apocrine carcinoma in $0.8 \%$ ( 1 patient), invasive papillary carcinoma in $0.8 \%$ ( 1 patient), malignant phyllodes tumor in $0.8 \%$ (one patient), malignant peripheral nerve sheath tumor in $0.8 \%$ (one patient), medullary carcinoma in $0.8 \%$ (one patient) and metaplastic carcinoma of the breast in $0.8 \%$ (one patient). Mixed types were found to be IDC+DCIS in 1.5\% (two patients), medullary carcinoma+IDC in $0.8 \%$ (onw patient), IDC+ILC in $0.8 \%$ (one patient), IDC+tubular carcinoma in $0.8 \%$ (1 patient) and ILC+IDC in $0.8 \%$ (one patient). In the IDC group, BCSs were performed on $45.5 \%$ of the patients, while MRMs were performed on $54.5 \%$. A statistically significant number of MRMs was performed in the IDC group $(p=0.037)$. There was no significant correlation between the other pathological types and choice of surgery.

\section{Discussion}

Radical mastectomy has been performed for a century with minor changes since it was defined by Halsted, depending on age, tumor size and tumor type. [1] Due to some unsatisfactory results of radical mastectomy operations, new surgical options have been researched. As an alternative to mastectomy, the BCS operations have also been carried out for almost 80 years. However, despite the satisfactory surgical results in the early periods, it is disappointing that the disease metastasizes. [7] This problem has been solved to a significant extent with the addition of RT to the treatment of patients undergoing BCS to prevent local recurrences.[8] Thus, having RT facilities in the center where the patients would undergo BCS has been a factor to be considered while planning the treatment of these patients. In an adjuvant RT study of breast cancer, which is one of the most extensive studies on this issue, there was no doubt that the application of radiotherapy to patients who had undergone BCS had results equivalent to mastectomy in terms of general survival and local control.[9,10] Thus, administering adjuvant RT has become the standard treatment for patients who have undergone BCS. However, studies found out that $15-30 \%$ of patients who had undergone BCS treatment did not receive adjuvant RT treatment despite the risk of ipsilateral relapse that may be caused by neglecting RT.[11-13] Travelling distance to treatment centers that have RT facilities may affect the postoperative breast irradiation process. It was reported that the rates of decisions to not receive
RT might be increased by the necessity of long-distance travelling during a process of 5-6 weeks for RT, problems in transportation and the costs to be created by them.[14] Lazovich et al.[11] conducted a study on breast cancer and revealed that living in a district where RT facilities were not available had a chance of lower than $50 \%$ about receiving RT after BCS. The rates of receiving RT following BCS significantly decreased by an increase in the travelling distance to the closest radiation treatment facilities.[14] Only $51 \%$ of women who were living at a 75 -mile distance or further from the closest facilities received radiotherapy, while $69 \%$ of them living at distances of 50-74.9 miles and $82 \%$ of them living at a distance of 50 miles received radiotherapy.[14] The closest facility with RT capacity to our region is $80 \mathrm{~km}$ (approximately 50 miles) far. Based on these data, we believe that transportation to facilities that have RT capacity may affect decisions on the RT. However, a similar study could not find a significant relationship between radiotherapy and travelling distance.[15]

In our study, we found that MRM operations were performed significantly more frequently at our hospital, which may be due to the tumor characteristics of the patients as well as our hospital's status as a newly established hospital. It is also possible that patients who would undergo BCS were more responsible and made an effort to explore other centers. Considering the numbers of MRMs and BCSs at our hospital, it was seen that there had been increasingly more BCSs after 2013. The reason why there was a smaller number of BCSs in 2018 was that when the data were collected, the year had not ended yet. The maximum number of BCSs that was performed was in 2017, whereas the maximum number of MRMs that was performed was in 2013. The increase in the BCS frequency after 2013 may be due to having RT facilities after the establishment of the Radiation Oncology Clinic, and given that multidisciplinary oncology councils have been held regularly since then.

In our study, the age range of the patients undergoing BCS was 40-45, while that of the patients undergoing MRM was 55-60 years. Breast cancer follows a more aggressive course in pre-menopausal patients and especially in patients under the age of 35.[16] Thus, some surgeons tend to suggest mastectomy in young patients more frequently.[17] However, it provides good local control with a wide conservative surgery in young patients ( $<35$ years). [18] In our study, given that the patients undergoing BCS were younger than the patients undergoing MRM may have caused aesthetic 
concerns, as well as tumor characteristics, to be more prevalent in young patients while choosing BCS.

It was found in our study that MRM was performed more in the breast cancer cases with left-sided lateralization, and BCS was performed more in the patients with right-sided lateralization. To our knowledge, this information has not been mentioned in the literature. It is asserted in general that left-sided breast cancer occurs more frequently. According to the Danish Cancer Registry, out of 4139 female breast cancer cases, 2117 cases had left lateralization, 1908 cases had right lateralization, 97 had bilateral lateralization, and lateralization was not specified in 17 cases.[19] Moreover, according to the United States Surveillance, Epidemiology, and End Results (SEER) [20] data, lateralization types of more than 250.000 breast cancer cases were examined according to sex, race, tumor stage, histology, diagnosis age, diagnosis year and estrogen receptor condition. The data, including all invasive and in-situ breast cancer cases, indicated that there were 5\% more cancer cases with left-sided lateralization in women, and the results obtained from other studies were confirmed.[21] However, it was revealed through the data of the same registry system that the proportions of women with breast cancer localized on the left side and the right side who were undergoing radiotherapy were similar among many patients in the categories of the stage, tumor location, age and ethnic origin. It was noted that the laterality of breast cancer plays very little role in determining who should undergo radiotherapy.[20] The reality that is known for the laterality of breast cancer is that, among women who undergo radiotherapy due to breast cancer, the mortality rate due to heart disease and ipsilateral lung disease increases in the patients with left-sided lateralization in comparison to the patients with right-sided lateralization. $[20,22,23]$ The reason for performing more MRMs on the left side of our study may be because cancer occurs more frequently on the left side. Considering our study in general, BCSs were performed on cases with good prognostic characteristics, and MRMs were performed on cases with poor prognostic characteristics. However, according to the literature, it is not foreseen that the prognosis of breast cancer localized on the left side may be worse at the time of diagnosis.[20] It was reported that what worsens the prognosis is cardiac and ipsilateral lung toxicity emerging after $10-15$ years due to left breast radiotherapy. The question that comes to mind here is: "Would it be possible that the cysts of the patients on the left breast were noticed later, and the cysts of the right breast were noticed earlier?"
Ultimately, the reason for the increased incidence of left-sided breast cancer in women or the reason why MRMs were performed more frequently on the left side, and BCSs were performed more frequently on the right side is not clear.

Tumor diameter that expresses the anatomical size of tumors is a good prognostic parameter for cancer patients. In TNM, the T stage is determined by presenting the numerical value of the tumor diameter at a certain interval. It was stated that, instead of the use of T this way (such as T1, T2, T3 and T4), its direct numerical value might be used.[24] In our study, when we compared the patients with tumors larger than 2.5 $\mathrm{cm}$ and the patients with tumors smaller than $2.5 \mathrm{~cm}$ concerning the types of surgery, we found that MRM was performed significantly more frequently on the patients with a tumor larger than $2.5 \mathrm{~cm}$. Moreover, we found that significantly more MRMs were performed as the $\mathrm{T}$ and $\mathrm{N}$ stages increased.

ER and PR positivity and negative values of $\mathrm{c}$ erbB-2 are generally indicators of good prognosis. [25] ER and PR positivity rates were respectively $55.0 \%$ and $62.5 \%$ in the patients undergoing BCS and $45.0 \%$ and $37.5 \%$ in the patients undergoing MRM. While there was no significant correlation between ER positivity and choice of surgery, it was found that significantly more BCSs were performed on the PR-positive patients. This may have been caused by the fact that PR positivity is a significantly better indicator of prognosis than ER.

Performing MRMs was almost significantly related to cases where c-erbB-2: +++. It is not surprising that MRMs were performed on the patients with c-erbB-2: +++ , considering that MRMs are performed on the patients with poor prognostic factors in general.

The patients' places of living were not found to be significantly correlated with tumor diameter or nodal stage. In contrast, Andersen et al.[26] stated that people living in rural areas lag behind the patients living in cities concerning early diagnosis of breast cancer. Comparatively, in our study, the rate of MRMs in the patients living in rural areas was higher, and the rate of BCSs in the patients living in cities was higher.

We found that there were not many patients admitted to our hospital from outside the province. Approximately $70 \%$ of the patients who were admitted to our hospital were from Rize. Artvin, the closest neighboring province, ranked the second about the number of patients that were admitted to our hospital from a different province. The patients who were admitted to our hospital from Artvin were found to undergo BCSs more 
frequently. In referrals from Rize, the rates of BCSs and MRMs were found to be almost evenly distributed.

The most common histological subtype of invasive breast cancer is IDC, which constitutes $70-80 \%$ of cases and ILC is the second most common histological subtype with a rate of 5-15\%.[27,28] DCIS constitutes about $12 \%$ of newly diagnosed breast cancers with an increasingly growing rate.[20] The most common pathological type in our study was found to be IDC (74.4\%). IDC was followed by ILC, tubular carcinoma and DCIS (the rates of frequency were $4.5 \%, 4.5 \%$ and $2.3 \%$, respectively). The DCIS rate in our study was found to be very low in comparison to other reports in the literature. When the relationship between pathological types and surgeries was considered, it was found that significant levels of MRMs were performed in the IDC group. This may be related to that IDCs are more common and have a worse prognosis. There was no significant correlation between the other pathological types and choice of surgery.

\section{Conclusion}

Most women with breast cancer are usually treated with RT following surgery. In general, worse prognostic factors in breast cancer may possibly require more extensive surgical treatments. In our study, as well as being younger, right-sided lateralization and living in cities were found to increase the frequency of BCSs, while left-sided lateralization and living in rural areas were associated with more MRMs that were performed. Having radiotherapy facilities at surgery centers should also be pointed out as a reason that increases the number of BCSs. In conclusion, the choice of surgical treatment is influenced by the characteristics of hospitals and other information about breast cancer. However, there is a need for more comprehensive studies to more clearly understand the data presented in this study, which was carried out with a small sample of patients.

Peer-review: Externally peer-reviewed.

Conflict of Interest: The authors have no conflicts of interest to declare.

Ethics Committee Approval: The authors declare that this research was conducted according to the principles of the World Medical Association Declaration of Helsinki "Ethical Principles for Medical Research Involving Human Subjects" (amended in October 2013).

Informed Consent: Informed consent was not obtained due to the retrospective nature of this study.
Financial Support: The authors declare that this study received no financial support.

\section{References}

1. Halsted WS. I. A Clinical and Histological Study of certain Adenocarcinomata of the Breast: and a Brief Consideration of the Supraclavicular Operation and of the Results of Operations for Cancer of the Breast from 1889 to 1898 at the Johns Hopkins Hospital. Ann Surg. 1898;28(5):557--76.

2. Fisher B, Fisher ER. Transmigration of lymph nodes by tumor cells. Science 1966;152(3727):1397-8.

3. Fisher B, Fisher ER. The interrelationship of hematogenous and lymphatic tumor cell dissemination. Surg Gynecol Obstet 1966;122(4):791-8.

4. Fisher B, Anderson SJ. The breast cancer alternative hypothesis: is there evidence to justify replacing it? J Clin Oncol 2010;28(3):366-74.

5. Cardoso F, Senkus E, Costa A, Papadopoulos E, Aapro $\mathrm{M}$, André $\mathrm{F}$, et al. 4th ESO-ESMO International Consensus Guidelines for Advanced Breast Cancer (ABC 4)†. Ann Oncol 2018;29(8):1634-57.

6. Franceschini G, Martin Sanchez A, Di Leone A, Magno S, Moschella F, Accetta C, et al. New trends in breast cancer surgery: a therapeutic approach increasingly efficacy and respectful of the patient. G Chir 2015;36(4):145-52.

7. Keynes G. Conservative Treatment of Cancer of the Breast. Br Med J 1937;2(4004):643-66.3.

8. Veronesi U, Luini A, Del Vecchio M, Greco M, Galimberti V, Merson M, et al. Radiotherapy after breastpreserving surgery in women with localized cancer of the breast. N Engl J Med 1993;328(22):1587-91.

9. Litière S, Werutsky G, Fentiman IS, Rutgers E, Christiaens MR, Van Limbergen E, et al. Breast conserving therapy versus mastectomy for stage I-II breast cancer: 20 year follow-up of the EORTC 10801 phase 3 randomised trial. Lancet Oncol 2012;13(4):412-9.

10. Fisher B, Anderson S, Redmond CK, Wolmark N, Wickerham DL, Cronin WM. Reanalysis and results after 12 years of follow-up in a randomized clinical trial comparing total mastectomy with lumpectomy with or without irradiation in the treatment of breast cancer. N Eng J Med 1995;333(22):1456-61.

11. Lazovich D, White E, Thomas DB, Moe RE. Underutilization of breast-conserving surgery and radiation therapy among women with stage I or II breast cancer. JAMA 1991;266(24):3433-8.

12. Young WW, Marks SM, Kohler SA, Hsu AY. Dissemination of clinical results: mastectomy versus lumpectomy and radiation therapy. Med Care 1996;34(10):1003-17. 
13. Farrow DC, Hunt WC, Samet JM. Geographic variation in the treatment of localized breast cancer. $\mathrm{N}$ Engl J Med 1992;326(17):1097-101.

14. Athas WF, Adams-Cameron M, Hunt WC, Amir-Fazli A, Key CR. Travel distance to radiation therapy and receipt of radiotherapy following breast-conserving surgery. J Natl Cancer Inst 2000;92(3):269-71.

15. Mann BA, Samet JM, Hunt WC, Key CR, Goodwin JM, Goodwin JS. Changing treatment of breast cancer in New Mexico from 1969 through 1985. JAMA 1988;259(23):3413-7.

16. Colleoni M, Rotmensz N, Robertson C, Orlando L, Viale G, Renne G, et al. Very young women $(<35$ years) with operable breast cancer: features of disease at presentation. Ann Oncol 2002;13(2):273-9.

17. van Nes JG, van de Velde CJ. The preferred treatment for young women with breast cancer--mastectomy versus breast conservation. Breast 2006;15 Suppl 2:S3-10.

18. Gentilini O, Botteri E, Rotmensz N, Toesca A, De Oliveira H, Sangalli C, et al. Breast-conserving surgery in 201 very young patients ( $<35$ years). Breast 2010;19(1):55-8.

19. Busk T, Clemmesen J. The frequencies of left- and rightsided breast cancer. British J Cancer 1947;1(4):345-51.

20. Darby SC, McGale P, Taylor CW, Peto R. Long-term mortality from heart disease and lung cancer after radiotherapy for early breast cancer: prospective cohort study of about 300,000 women in US SEER cancer registries. Lancet Oncol 2005;6(8):557-65.

21. Weiss HA, Devesa SS, Brinton LA. Laterality of breast cancer in the United States. Cancer Causes Control 1996;7(5):539-43.
22. Cuzick J, Stewart H, Rutqvist L, Houghton J, Edwards R, Redmond C, et al. Cause-specific mortality in long-term survivors of breast cancer who participated in trials of radiotherapy. J Clin Oncol 1994;12(3):447-53.

23. Favourable and unfavourable effects on long-term survival of radiotherapy for early breast cancer: an overview of the randomised trials. Early Breast Cancer Trialists' Collaborative Group. Lancet 2000;355(9217):1757-70.

24. Köksal M, Doğan S, Öztürk F, Cücer N. Investigation of the Relationship Between the Largest Tumor Size, AgNOR area and AgNOR Count on Breast Cancer Cells. Journal of Natural and Applied Sciences 2016;32(2).

25. Bauer KR, Brown M, Cress RD, Parise CA, Caggiano V. Descriptive analysis of estrogen receptor (ER)-negative, progesterone receptor (PR)-negative, and HER2-negative invasive breast cancer, the socalled triple-negative phenotype: a population-based study from the California cancer Registry. Cancer 2007;109(9):1721-8.

26. Andersen LD, Remington PL, Trentham-Dietz A, Robert S. Community trends in the early detection of breast cancer in Wisconsin, 1980-1998. Am J Prev Med 2004;26(1):51-5.

27. Li CI, Anderson BO, Daling JR, Moe RE. Trends in incidence rates of invasive lobular and ductal breast carcinoma. JAMA 2003;289(11):1421-4.

28. Gültekin M, Boztaş G. Türkiye Kanser İstatistikleri, Sağlık Bakanlığı, Türkiye Halk Sağlığı Kurumu. Ankara: 2014. 